\author{
Military Technical College \\ Kobry El-Kobbah, \\ Cairo, Egypt.
}

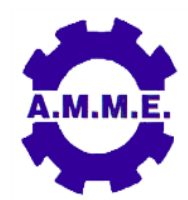

\title{
EARTH OBSERVATION SATELLITES OPTIMIZATION; SURVEY AND ANALYSIS
}

\author{
A. M. Abbas ${ }^{*}$, K. T. Meselhey ${ }^{*}$ W. S. Mogheth ${ }^{*}$ and A. A.Omer
}

\begin{abstract}
The problem of earth observation satellites planning and scheduling contains many objectives and constraints. The objectives include downloaded data, profit of scheduled tasks and quality (weight) values. The constraints are related to energy, data, targets (tasks) and ground stations. Many optimization methods have been used to optimize this problem such as dynamic programming (DP), simulated annealing (SA), ant colony optimization (ACO), genetic algorithm (GA) and constraint programming approach (CPA). Each reported research used a harmonized combination of objective(s), constrains, and optimization techniques. The presented work investigates the anatomy of this optimization problem. It is also analyzes the findings of the previous relation between the optimization problem elements and gaps in past research. Furthermore shows that there is a gap between data's objectives (maximizing the total amount of downloaded data) and the targets' constraints (observation of tasks, scheduling and consecutive observation). The most widely used optimization methods are dynamic programming and heuristic algorithms, respectively. Moreover, the most widely objectives are those relative to profit. Few discussions are also presented concerning the constraints which involve data rate, range of ground stations and capacity of ground stations.
\end{abstract}

\section{KEY WORDS}

Objective functions, Constraints, Optimization, Optimization methods, Earth, observation, Satellites, EOS.

\footnotetext{
* Egyptian Armed Forces.
} 


\section{INTRODUCTION}

Planning and scheduling problem of Earth Observation Satellite (EOS) has been discussed in many papers. Each paper has its own specific conditions. These conditions include types of satellite's operation (imaging, downloading or both) [1], maneuverability of satellites (not agile or agile) $[2,3,4]$, number of tasks (single or multi) [2], number of satellites (single or constellation) [5, 6, 7, 8, 9, 10, 11, 12, 13], and number of ground stations (single or multi) $[6,7]$.

Satellite earth observation is considered a platform which equipped with imaging instruments and orbits the earth for taking images of specific areas according to users' requests [5]. In the introduction, the meaning of satellite, satellites' applications, orbits, launch window, payload, remote sensing, earth observation, ground system and space system will be discussed.

\section{Satellite Definition}

The satellite is defined generally according to NASA as a moon, planet or machine that orbits a planet or star. This word refers to a machine that is launched into the space and move around the earth or another body in space. [14]

\section{Applications of Satellites}

The following list the different applications of satellites; such as [15]:

1) Weather Forecasting

Monitor the climatic conditions of the earth by taking images of earth, predict disasters and monitor the changes in sea state, earth's vegetation and ocean color.

2) Radio and TV Broadcast

Make hundreds of channels across the globe and broadcast news, live matches and world-wide radio services.

3) Military Satellites

Collect intelligence, the character of the satellite if it is civilian or military can be defined by the kind of satellite's payload.

4) Navigational Satellites

Precise the location world-wide and also may be in the range of some meters.

5) Global Telephone

Set up international telephone backbone but fiber optics is used instead of communication satellite.

6) Connecting Remote Area

Supply coverage for the internet or telephone network.

7) Global Mobile Communications

Increase the coverage area for mobile communication.

The third application is considered the most interesting application as it is mainly depends on taking photos through its payload (camera).

\section{Orbits and Satellites}

The orbit is the trajectory or path of the spacecraft. A trajectory or an orbit for a 
satellite refers to its path through the space. There are types of orbits such as parking orbit, transfer orbit, final mission orbit and disposal orbit. Astrodynamics is the study of satellite's orbit or trajectory [16].

Kepler [16] has published two laws of planetary/satellite motion in 1609. The first one is that the orbit of each planet/satellite has an ellipse with the sun at one focus, whereas the second one is that the line joining the planet to the sun is sweeping out equal areas in equal times.

Today, all satellites get into orbit by riding on a rocket or riding in the cargo bay of a space shuttle [17].The following points describe orbits in detail:

1) Key Parameters of an elliptical orbit

$r$... Position vector of the satellite relative to the earth's center

$V$... Velocity vector of the satellite relative to the earth's center

$a$... Semimajor axis of the ellipse

$b$... Semiminor axis of the ellipse

$c$... The distance from the center of the orbit to one of the focuses

$v$... The polar angle of the ellipse measured in the direction of satellite's motion from the perigee direction to the position vector, also called true anomaly

$r_{A} \ldots$ Radius of apogee, the distance from the center of the earth to the farthest point on the ellipse

$r_{P} \ldots$ Radius of perigee, the distance from the center of the earth to the closest point approach to the earth

Fig. 1 illustrates the above parameters.

2) Orbital Elements

They are considered a completely description for the orbit.

3) Orbital Period

It refers to the time at which the satellite takes between two successive ascending nodes.

4) Perturbations

Any forces perturb the satellite away from its nominal orbit are considered perturbations. These perturbations occur due to non-spherical earth, atmospheric drag and solar radiation.

\section{Launch Window}

The launch window is the time at which the site for launch on the earth's surface rotates through the orbital plane or it is a particular period of time in which it will be easier to put the satellite necessary to perform its intended duty/mission. For the existence of the launch window, the site for launch must pass through the orbital plane. The launch time depends on the inclination of satellite orbit, right ascension of ascending node and latitude and longitude of the launch site $[16,17]$.

\section{Payload}

The payload is the combination of software and hardware on the spacecraft which interacts with the subject to accomplish the objectives of the mission, where the subject of the mission is the thing which interacts with or is sensed by the payload. The payload is the main reason for the spacecraft. The purpose of other components 
of the spacecraft is to protect the payload and make sure that its points are in the right direction [16].

\section{Remote Sensing}

Any observation that a spacecraft makes without directly contacting the required object is considered remote sensing. Imaging the surface of the earth or providing early warning of a ballistic missile launch is all remote sensing missions [16].

\section{Earth Observation}

Earth coverage is the part / area of the earth that a satellite's payload or antenna can see over an extended period or at one instant. In evaluating coverage, two critical differentiations must be taken in consideration. The first one is the instantaneous field of view, called FOV or footprint, is the actual area that can be seen by the payload or antenna at any moment. In contrast, the access area is the total area on the ground that could be seen potentially at any moment by turning the satellite or the payload. The second one is between the area that can be seen at any moment and the rate at which new area comes into view as spacecraft and payload move [16].

Nadir is a point on the surface of the earth which has the shortest distance from the satellite to the earth's surface. The projection of the satellite's orbit on the surface of the earth is called the ground track. Swath of a satellite is the width of the area on the ground surface which is imaged by the sensor during a single pass as shown in Fig. 2. The common area on consecutive images along flight direction is called overlap. The elapsed time between two successive views of the same area by a satellite is called revisit period [18].

The elevation angle of a satellite is the angle between the satellite and the local horizon at the target point as seen in Fig. 3. It varies with time as the satellite moves [19].

The observable size of an earth's area from a satellite depends on its orbital altitude. The observable area is compared in Fig. 4 for satellites at two different altitudes: the satellite at the lower altitude sees a smaller area than the one at the higher altitude. Note that the observable area also describes the area on the Earth that can see the satellite [18].

Earth observation satellites are considered platforms which equipped with imaging instruments that orbit the earth to take images of specific areas at users' requests. Satellite-based cameras are characterized by broad coverage, without limitation on the national boundaries and long duration. These EOSs play important roles in remote sensing of natural resources, cartographic mapping, evaluation of natural disasters and military reconnaissance [20].

There is a new generation of EOS with three degree of freedom, such as the French Pleiades. This generation is called Agile EOS (AEOS). At this new generation, all instruments are fixed on the satellite and the whole satellite can move on the three axes (roll, pitch and yaw). Because of these new capabilities of satellite, the observing time windows become much longer and there are too many ways to 
observe a target area, since starting times of observations are free which bring also different image quality. As a result, agile satellites can observe much more tasks than non-agile satellite but there will be more difficulties to the selection and scheduling of AEOS observations since search space is become larger [2].

When a satellite flies over the target area, its camera is opened to take required image. Because of the high speed of the satellite and the time taken for imaging process, a strip that covers the target will be produced. Each target is thus associated with a special time window. For some targets, there is a need to roll the satellite's camera to take the image due to the restriction of satellite's field of view [20].

\section{Ground System and Space Segment}

The spacecraft and their payloads are considered the space segment. The ground stations and control centers are considered the ground system and it may be fixed or mobile. The ground system supports the space segment and relays mission data to users which is generated by onboard instruments and received from the spacecraft [16]. Each ground station may have multiple download options from which to choose, where each option is defined by a combination of values for data rate, efficiency and energy utilization [21].

To support the space segment, the ground system must command and control it, monitor its behavior, track it to determine orbital position and determine spacecraft attitude from sensor information. The spacecraft and its instruments / payloads have been controlled by transmitting command data from the ground system to the spacecraft [16].

There are three types of control centers which are found within ground systems. The first one is the space operations control center (SOCC) which monitors and commands the spacecraft bus and common systems (onboard instruments or payloads) and it also analyzes spacecraft telemetry and mission data from instruments which may affect the spacecraft attitude and dynamics. The second one is the payload operations control center (POCC) which analyzes telemetry and mission data from onboard payload instruments and issues commands to these instruments. The third one is the mission control center (MCC) which plans and operates the entire space mission including the configuration and scheduling of resources for both space and ground system [16].

In this paper, it touches on the problem of earth observation satellites planning and scheduling especially, its contents of objectives and constrains. It contains a wide survey for gathering information about objectives, constraints and optimization techniques that belongs to our problem. The objectives may be divided into three portions (downloaded data, profit of scheduled tasks and quality/weight values) and constraints may be divided related to many factors (energy, data, targets/tasks and ground stations). Many optimizations techniques have been used for solving this problem. A table is established showing the relation between them and it will be useful for any researchers who keen about this problem. 
After the introduction, five sections will be discussed. Second section contains categorization for the objective functions of the planning and scheduling problem for earth observation satellites. Third section contains also a categorization for the constraints of that problem. At the fourth section, a categorization for the optimization methods has been used for solving that problem. At the end, a conclusion drawn from the presented work is introduced.

\section{PROBLEM CATEGORIZATION ACCORDING TO OBJECTIVE FUNCTIONS}

The objective functions of our problem are categorized to many factors as follows:

\section{Downloaded Data}

The amount of downloaded data needs to be increased as possible in case of receiving from a satellite or more than one. The amount of un-downloaded data needs to be decreased as possible in case of it is still onboard the satellite. It is clear that downloaded data can be taken as an important factor which has an effect in creating objective functions.

\section{Maximize the total amount of data received over the planning horizon}

There is a necessary to maximize the amount of downloaded data from a single satellite orbiting the earth to ground stations. The acquisition rates for satellite's energy and data may vary over time. For example, the line of sight of the solar panels relative to the sun has an effect on the energy's collection whereas the satellite is in view of the specific target has an effect on the data's collection. The limitations for the permitted stored data and energy cause discontinuities in the system dynamics which result in non-linear optimization problem. To overcome this deficiency, the problem is divided into a finite set of time intervals to approximate the continuous time dynamics [6]. The following equation represents this objective function.

Objective Function $=\operatorname{Max} \sum_{i \in I} \sum_{o \in O} \eta_{i o} q_{i o}$

where:

$I \ldots$ Set of intervals, $\forall i \in I$

$O$.... Set of download options

$O_{i o} \ldots$. The subset of download options available during interval ( $\left.{ }^{i}\right), \forall O_{i} \subseteq O$

$q_{i o} \ldots$. The amount of data downloaded during interval $(i)$ using option ( $\left.{ }^{o}\right), \forall o \in O_{i}$

$\eta_{i o} \ldots$.... The efficiency during interval $(i)$ when downloading using option $\left({ }^{o}\right)$

Maximize the total amount of data that is downloaded from each satellite during the planning horizon

In case of scheduling the downloading of data from a constellation of satellites to multiple ground station, it is called a multiple satellite multiple ground station scheduling problem (MMSP). At this problem, there is a need to maximize the total amount of downloaded data which have been downloaded from each satellite to match the (suitable) ground station according to the visibility zones for each ground 
station [7]. The following equation represents this objective function.

Objective Function $=\operatorname{Max} \sum_{s \in S} \sum_{i \in I} \sum_{g \in G} \eta_{i g} q_{s i g}$

where:

$S$... Set of satellites, $\forall s \in S$

$G$... Set of ground stations, $\forall g \in G$

$I$... Set of time intervals, $\forall i \in I$

$\eta_{i g} . .$. The efficiency (fraction of downloaded data successfully received by the ground station) during interval (i) when downloading to ground station (g)

$q_{\text {sig }} .$. The amount of data downloaded by satellite (s) during interval (i) to ground station $(\mathrm{g})$

Collecting the largest amount of prioritized imagery and continually lowering the amount of un-downloaded data onboard the satellite

The goal of collecting the largest amount of prioritized imagery and continually lowering the amount of un-downloaded data onboard the satellite is achieved here by Mixed Integer Linear Programming (MILP). This goal is considered a multi objective gathering objective functions for downloaded data and profit. This method touched on both image and downlink opportunities simultaneously. For setting the storage penalty, the waiting time that image data spends on-board the satellite before it gets downlinked will be minimized. The penalty is proportional to the amount of data and time duration; it will be stay on-board [9]. The following equation represents this objective function.

Objective Function $=\operatorname{Min}_{x_{i}} \sum_{i \in S_{\text {all }}}\left(-c_{i} x_{i}+\alpha d_{i} y_{i}\right)$

where:

$S_{\text {all } \ldots . . . S e t}$ of all opportunities (Image and downlink opportunities)

$i$... An opportunity,

$x_{i} \ldots$ A Boolean variable indicating inclusion in the schedule,

$y_{i} \ldots$ Amount of onboard data at end time of opportunity $(i)$

$c_{i}$... Priority weight for opportunity $(i)$

$c_{i} \succ 0 \ldots$ For imaging opportunity $\left({ }^{i}\right)$, how important capturing that image is

$c_{i}=0 \ldots$ For all downlink opportunities

$d_{i} \ldots$ A penalizing cost of onboard data storage for opportunity $(i)$

$\alpha \ldots$ A parameter that controls the relative importance of imaging versus downlinking, it is set empirically

\section{Profit of Scheduled Tasks}

The number of selected tasksltargets needs to be increased as possible. That number and the priority of those tasksltargets in addition to completing dynamic tasksltargets as possible may be considered the profit of scheduled tasksltargets. It 
is clear that the profit of scheduled tasksltargets can be taken as an important factor which has an effect in creating objective functions.

\section{Maximize the total priority of selected tasks}

Observation request from users is considered to be a task (Take a photograph for a specific area on the earth by a satellite). Agile Earth Observation Satellites (AEOS) problem consists of selecting which tasks to perform, determining which time windows to be used and the starting time of a task in a time window. As a result, it is considered to be over-constrained and all tasks can't be performed. The number of total task sequences allocated on each satellite is uncertain before constructing a complete solution; so the number of sequences is set to be equal to the number of tasks. The number of window index is set to be equal to the maximum number of alltime windows for all tasks because there is uncertain number of available time windows for each task on a satellite (Each task has its own number for time windows and this number varies from to task to another one) [3]. The following equations represent this objective function.

Objective Function $=\operatorname{Max} \sum_{m=1}^{k} \sum_{s=1}^{w} \sum_{b=1}^{n} \sum_{j=1}^{n} O_{j} X_{j b s m}$

Objective Function $=\operatorname{Max} \sum_{i=1}^{n} x_{i} \cdot P_{i} \cdot$ Area $_{i}$

where:

$j \ldots$ Task index, $j=1,2 \ldots \mathrm{n}$

$b$... Sequence index, $b=1,2 \ldots \mathrm{n}$

$m$... Satellite index, $m=1,2 \ldots \mathrm{k}$

$s \ldots$ Window index, $s=1,2 \ldots \mathrm{w}$

$O_{j} \ldots$ Priority of task $\left({ }^{j}\right)$ determined by decision makers ${ }^{X_{j b s m}} \ldots$ Decision variable, $X_{j b s m} \in\{0,1\}$

$X_{j b s m} \ldots$ If task $(j)$ is assigned at the $\left(s^{\text {th }}\right)$ window with the $\left(b^{\text {th }}\right)$ sequence scheduled on satellite $(m) ; X_{j b s m}=0 \quad$ otherwise;

$x_{i}$... Decision variable $\left(x_{i}=1\right.$ ), If task $(i)$ is included on the schedule $\&^{x_{i}}=0$, If it isn't included on the schedule)

$P_{i} \ldots$ Priority of task $(i)$

Area $_{i} \ldots$... Area of task $(i)$

Maximize the profit of satellite (Summing up priorities of observed tasks)

More than one satellite may fulfill it and each satellite owns its several observation windows. To define these observation chances, Meta-tasks will be introduced. A task may create several meta-tasks. Only one of its meta-tasks is selected to observe. Combined task is a combination of meta-tasks. Combined observation ensures that satellites fulfill more tasks, it is also good for satellite's gesture keeping, reducing slewing times of the satellite and unnecessary power consumption will be cut down. Multi Satellites Scheduling Problem (MSSP) for satellites with limited slewing ability 
has been formulated. Here, this equation is summing up priorities of observed tasks to get the profit of the satellite [10]. The following equation represents this objective function.

Objective Function $=\operatorname{Max} \sum_{i=1}^{N_{C}} \sum_{j=1}^{N_{t}} f(i, j) \cdot P_{j}$

where:

$f(i, j)$...Boolean value, which is used to mark whether $\operatorname{task} T_{j}$ is contained in combined task $\mathrm{Obs}_{i}$

$N_{C} \ldots$ Number of combined tasks

$N_{t}$...Number of tasks

$P_{j} \ldots$ Priority of observed task

\section{Completing dynamic tasks as many as possible \& Maximize the observation income}

Dynamic tasks are emergency tasks. They should be completed in dynamic scheduling immediately when they are added to the task set. The optimal observation income always is considered the main goal for scheduling process. It is difficult to calculate the optimum due to the complexity of the problem and the limitation of computing ability. So, decision makers often have to accept a satisfied near optimal solution. There may be several visible time windows for every task observed by each satellite [20].

Here, there are two optimization objectives. The first one is completing as many dynamic tasks as possible which is considered important for the emergency scheduling. The second one is to maximize the observation income which is considered the common objective for all imaging scheduling [20]. The following equations represent those objective functions.

Objective Function $=\operatorname{Max} \sum_{i=1}^{d_{n}} \sum_{j=1}^{m} \sum_{k=1}^{\left|W_{i}{ }^{j}\right|} x_{i, k}^{j}$

Objective Function $=\operatorname{Max} \sum_{i=1}^{n} \sum_{j=1}^{m} \sum_{k=1}^{\left|W_{i}{ }^{j}\right|} x_{i, k}^{j} \cdot C_{i}$

Objective Function $=\operatorname{Max} \sum_{j \in j_{1}} w_{j} \sum_{P \in O T W_{j}} x_{P}+\sum_{j \in j_{2}} w_{j} f\left(\Pi_{j} / S_{j}\right)$

where:

$d_{n} \ldots$ The number of dynamic tasks

$m$... The number of satellites

$W_{i}{ }^{j} \ldots$ The set of time windows observed by satellite $(j)$ for task $(i)$

$\left|W_{i}^{j}\right| \ldots$ The number of visible time windows of task $(i)$ observed by satellite $(j)$

$n \ldots$ The number of all tasks

$x_{i, k}^{j} \ldots$ Decision (Boolean) variable 
$x_{i, k}^{j}=1 \ldots$ If the task $(i)$ can be observed by the satellite $(j)$ in the time window;

$x_{i, k}^{j}=0$ otherwise

$C_{i} \ldots$ The observed income of task $(i)$

$w_{j} \ldots$ The reward of target

$x_{P} \ldots$ Binary variable for observation time window $P \in O T W$, representing whether the time window $P$ is adopted as an observation

$\Pi_{j} \ldots$ Nonnegative continuous variable for polygon $j \in J_{2}$, denoting the summed contribution area of all the observed time windows $P \in O T W_{j}, j \in J_{2}, x_{P}=1$, deducting the overlapped area among the time windows

$S_{j} \ldots$ Denotes the total area of polygon $j \in J_{2}$

\section{Quality (Weight) Values}

The covered area and the resolution of the images need to be increased as possible. That number and the priority of those tasksltargets in addition to completing dynamic tasks ltargets as possible may be considered the profit of scheduled tasksltargets. It is clear that the profit of scheduled tasksltargets can be taken as an important factor which has an effect in creating objective functions.

\section{Maximize the quality values}

There are agile satellites and different targets with different rolling angles. It is considered a combinatorial optimization problem (Optimally planning the images' acquisition and assigning them to the satellites of a constellation). Quality values are assigned as functions of the area that strips cover and the distortion of the image. These quality values are used to determine the differences among the strips that are available to be imaged. A directed graph has been used as shown in Fig.5 [11]. The following equations represent our objective function which is to maximize the quality values.

Objective Function $=\operatorname{Max} \sum_{i=1}^{N} q_{i j}$

Objective Function $=\operatorname{Max} \sum_{j \in N} \sum_{C \in M^{j}} w_{j} x_{j}^{C}$

where:

Node $(i) \ldots$ A pass over area of interest (AOI)

$V \ldots$ Set of Nodes, $\forall i \in V \& V=\{1, \ldots \ldots \ldots, N\}$

$j$... Edges that belong in a selected path

$q_{i j} \ldots$ Quality values, assigned to the edges, as functions of the area that they cover and the distortion of the image

$w_{j} \ldots$ The weight of image $(j)$

$x_{j}^{C} \ldots$ Denotes by a $0-1$ variable that image $(j)$ that can be taken by a camera $(C)$

$M^{j} \ldots$ The subset of cameras that can take image $(j)$

$N$... The set of images to be taken 


\section{Maximize the Weight values}

The satellite data transmission scheduling problem has been formulated to maximize the weight values meaning the importance degree of target weight and satellite resolution weight; data requested satisfied degree and time urgency. Before this formulation, some assumptions are considered to simplify the problem such as; a satellite can only perform one mission at time, a Tracking and Data Relay Satellite System (TDRSS) satellite can serve multiple satellites at the same time and a ground station and mobile station can only serve one satellite in one access window [1]. The following equation represents the objective function which is to maximize the weight values.

Objective Function $=\operatorname{Max} P_{1}\left(\sum_{i \in T G T} w_{i} r_{i} x_{i}\right)+P_{2}\left(\sum_{o t \in \in O T W} x_{\text {otw }}\right)+P_{3}\left(\sum_{i \in T G T} x_{i} / \sum_{i \in T G T}\left(t_{i}-a_{\text {scene }}\right) x_{i}\right)$

where:

$P_{1} \ldots$ Important degree of target weight and satellite resolution weight

$P_{2} \ldots$ Important degree of data requested satisfied degree

$P_{3} \ldots$ Important degree of time urgency

TGT ... Target set

$x_{i} \ldots$ Binary variable for target $i \in T G T$ representing whether target $(i)$ is arranged

OTW ... Observation time window set

$r_{i} \ldots$ Nonnegative integer variable representing the maximum satellite resolution weight for target $i \in T G T$

$w_{i} \ldots$ Nonnegative integer variable representing the important weight for target $i \in T G T$

$x_{\text {otw }} \ldots$ Binary variable for observation time window otw $\in$ OTW representing whether time window otwis adopted as an observation

$t_{i} \ldots$ The time of a user receiving data of target $(i)$

$a_{\text {scene }} \ldots$. The start time of the scene

\section{CONSTRAINTS}

There are many constraints which can effect on our problem and they can be categorized related to many factors as follows:

\section{Energy}

Energy constraints enforce that the amount of energy stored at the beginning of each interval is within battery buffer limits. [6, 7]; this can be expressed by Eqns.(13) and 14).

$$
\begin{aligned}
& e_{\text {min }} \leq e_{i} \leq e_{\text {max }} \\
& e_{\text {min }} \leq e_{s i} \leq e_{\text {max }}
\end{aligned}
$$

where:

$I$.... Set of intervals, $\forall i \in I$ 
$e_{i} \ldots$. The amount of energy available at the beginning of interval $(i)$

$e_{\max } \ldots .$. The maximum allowable amount of energy to be stored in the battery

$e_{\min }$.... The minimum allowable amount of energy to be stored in the battery

$S$.... The set of satellites, $\forall s \in S$

$e_{s i} \ldots$. The amounts of energy available for satellite $(S)$ at the beginning of interval $(i)$

\section{Data}

\section{Data buffer limits}

a- Data constraints enforce that the amount of data stored at the beginning of each interval is within data buffer limits [6,7]; this can be expressed by Eqns. (15) and (16).

$$
\begin{aligned}
& d_{\text {min }} \leq d_{i} \leq d_{\text {max }} \\
& 0 \leq d_{s i} \leq d_{\max }
\end{aligned}
$$

where:

$I$.... Set of intervals, $\forall i \in I$

$d_{i} \ldots$. The amount of data available at the beginning of interval $(i)$

$d_{\max } \ldots$. The maximum allowable data stored in the data buffer

$d_{\min } \ldots$. The minimum allowable data stored in the data buffer

$S \ldots$. Set of satellites, $\forall s \in S$

$d_{s i} \ldots$. The amounts of data available for satellite $(S)$ at the beginning of interval $(i)$

b- Data constraints enforce the satellite to use one option at most during each time interval in case of downloading [6]; this can be expressed by Eqn. (17).

$$
\sum_{O \in O_{i}} x_{i o} \leq 1
$$

where:

$I \ldots$ Set of intervals, $\forall i \in I$

$O \ldots$... Set of download options

$O_{i} \ldots$. The subset of download options available during interval $(i), \forall O_{i} \subseteq O$

$x_{i o} \ldots$. The binary value representing the decision of whether to download using option $(O)$ during some portion of interval $(i)$

\section{Time of each interval and data rate}

Data constraints enforce that the data can only be downloaded using the chosen option for any given interval and the amount of downloaded data is limited by the interval's time and the chosen data rate. [6, 7]; they are represented by Eqns. (18) and (19).

$$
q_{\text {io }} \leq \Delta t_{i} \phi_{i o} x_{i o}
$$




$$
q_{\text {sig }} \leq t_{i} \phi_{i g} x_{s i g}
$$

where:

$I$.... Set of intervals, $\forall i \in I$

$\Delta t_{i} \ldots$. The duration of time interval $(i), \Delta t_{i}=t_{i+1}-t_{i}$

$\phi_{i o} \ldots$. The data rate associated with downloading during interval $(i)$ using option $(O)$

$x_{i o} \ldots$. The binary value representing the decision of whether to download using option $(O)$ during some portion of interval $(i)$

$q_{i o} \ldots$. The amount of downloaded data during interval $(i)$ using option $(O)$

$S$.... Set of satellites, $\forall s \in S$

$G$.... Set of ground stations, $\forall g \in G$

$t_{i} \ldots$. The duration of interval $(i)$

$\phi_{i g} \ldots$. The data rate associated with downloading data to ground station $(g)$ during interval $(i)$

$x_{s i g} \ldots$. The continuous variable representing the percentage of interval $(i)$ during which satellite $(s)$ downloads to ground station $(g)$

$q_{s i g} \ldots$. The amount of downloaded data by satellite $(s)$ during interval $(i)$ to ground station $(g)$

\section{Capacity}

a- Capacity constraints ensure that the memory consumption of the scheduled tasks cannot exceed the memory capacity for each orbit [11, 12, 13, 21, 25, and 28]; they are represented by Eqns. (20) and (21).

$$
\begin{aligned}
& \sum_{i \in T} \sum_{\substack{j \in T \cup\{n+1\} \\
i \neq j}} x_{i j}^{k}\left(\omega e_{i k}-\omega s_{i k}\right) m_{k} \leq M_{k} \\
& \sum_{i=1}^{N} m_{i j} \leq M
\end{aligned}
$$

where:

$T$.... Set of tasks, $\forall T=\{1, \ldots ., n\}, \forall i \in T$

$i, j \ldots$. Task index, $i, j \in T \cup\{0, n+1\}$ is the immediate predecessor of task ( $j$ )

$O$.... Set of orbits, $\forall O=\{1, \ldots . ., m\}$

$k$.... Orbit index, $\forall k \in O$

$\left[\omega s_{i k}, \omega e_{i k}\right] \ldots$. Time window of observation of task $(i)$ on orbit $(j)$

$m_{k} \ldots$. Memory consumption for each unit time of observation orbit $(k)$

$M_{k} \ldots$... Memory capacity of orbit $(k), \forall k \in O$

$x_{i j}^{k} \ldots$ Decision variable, $x_{i j}^{k}=1$ If both tasks $(i),(j)$ are scheduled on orbit $(k)$ and task

$(i)$ is the immediate predecessor of task $(j) \quad$; Otherwise $x_{i j}^{k}=0$

$M$.... The total on board memory

$N$.... The number of passes over an area of interest (AOI)

$m_{i j} \ldots$. Memory consumption 
b- Capacity constraints ensure that the total capacity and working time of all tasks on each satellite do not violate the total satellite capacity and total working time $[3,22]$; it is expressed by Eqns. (22) and (23).

$$
\begin{gathered}
\sum_{j=1}^{n} \sum_{b=1}^{n} \sum_{s=1}^{w} c_{j} x_{j b s m} \leq C_{m} \\
\sum_{j=1}^{n} \sum_{b=1}^{n} \sum_{s=1}^{w} P_{j} \cdot x_{j b s m} \leq P_{m}
\end{gathered}
$$

where:

$j \ldots .$. Task index, $j=\{1,2, \ldots \ldots ., n\}$

$b$.... Sequence index, $b=\{1,2, \ldots \ldots ., n\}$

$s \ldots$. Window index, $s=\{1,2, \ldots \ldots ., w\}$

$m$.... Satellite index, $m=\{1,2, \ldots \ldots ., k\}$

$C_{m} \ldots$. The total memory storage capacity of satellite $(m)$

$c_{j} \ldots$. Memory capacity of task $(j)$

$P_{m} \ldots$. The total available consecutive working time of satellite $(m)$

$P_{j} \ldots$. Working time of task $(j)$

$x_{j b s m} \ldots$ A decision/Boolean variable representing if task $(j)$ is assigned at the window index with the $b^{\text {th }}$ sequence scheduled on satellite $(m)$, or not.

\section{Tasks (Targets)}

\section{Observation of tasks}

Tasks constraints ensure that each task will be observed at most once [10, 12, 20, $21,22,23,24,25,26,28$, and 29]. This constraint can be represented by Eqns. (24), (25), (26) and (27).

$$
\begin{aligned}
& \sum_{\substack{j \in T \\
i \neq j}} \sum_{k n+1\}} x_{i j}^{k} \leq 1 \\
& \sum_{k \in O} x_{i k} \leq 1 \\
& \sum_{i=1}^{N_{C}} f(i, j) . P_{j}=1 \\
& \text { where: } \\
& T \ldots . \text { Set of tasks, } \forall T=\{1, \ldots ., n\}, \forall i \in T \\
& i, j \ldots . \text { Task index, } i, j \in T \cup\{0, n+1\} \text { is the immediate predecessor of task }(j) \\
& O \ldots . \text { Set of orbits, } \forall O=\{1, \ldots ., m\} \\
& k \ldots . \text { Orbit index, } \forall k \in O
\end{aligned}
$$


$x_{i j}^{k} \ldots$ Decision variable, $x_{i j}^{k}=1$ If both tasks $(i),(j)$ are scheduled on orbit $(k)$ and task $(i)$ is the immediate predecessor of task $(j)$; Otherwise $x_{i j}^{k}=0$

$x_{i k} \ldots$ A Decision variable, $x_{i k}=1$ If both tasks $(i)$ is allocated to orbit $(\mathrm{k})$; Otherwise $x_{i k}=0$

$f(i, j) \ldots$ Boolean value, which is used to mark whether task $(i)$ is contained in combined task $\mathrm{Obs}_{i}$

$P_{j} \ldots$ Priority of observed task

$N_{C} \ldots$ Number of combined tasks

$$
\sum_{j=1}^{|S|} \sum_{k-1}^{\left|W_{i}{ }^{j}\right|} x_{i k}^{j} \leq 1
$$

where:

$S$.... Set of satellites, $\forall j \in S$

$W_{i}^{j} \ldots$. The set of time windows observed by satellite $(j)$ for task $(i), \forall k \in W_{i}^{j}$

$x_{i k}^{j} \ldots$. The decision/Boolean variable representing if the task $(i)$ can be observed by satellite $(j)$ in the $k^{\text {th }}$ time window or not

Tasks' constraints determine the starting time of a specific sequence on a specific satellite, which is equal to or larger than the begin time for the available window of the task processed in the current sequence or the completion time of the previous sequence [3]. This constraint can be represented by Eqns. (28) and (29).

$$
\begin{aligned}
& \sum_{j=1}^{n} \sum_{s=1}^{w} B\left(w_{j s m}\right) \cdot x_{j b s m} \leq S_{b m} \\
& C_{(b-1) m} \leq S_{b m}
\end{aligned}
$$

where:

$j \ldots .$. Task index, $j=\{1,2, \ldots \ldots ., n\}$

$b$.... Sequence index, $b=\{1,2, \ldots \ldots ., n\}$

$s \ldots$. Window index, $s=\{1,2, \ldots \ldots ., w\}$

$m$.... Satellite index, $m=\{1,2, \ldots \ldots ., k\}$

$S_{b m} \ldots$. The starting time of the $b^{\text {th }}$ sequence scheduled on satellite $(m)$

$C_{(b-1) m} \ldots .$. The completion time of the $(b-1)^{\text {th }}$ sequence scheduled on satellite $(m)$

$w_{j s m} \ldots$. The $s^{\text {th }}$ time window of task $(j)$ on satellite $(m)$

$B\left(w_{j s m}\right) \ldots$. The begin time of time window $w_{j s m}$

$x_{j b s m} \ldots$. A decision/Boolean variable representing if task $(j)$ is assigned at the $s^{\text {th }}$ window index with the $b^{\text {th }}$ sequence scheduled on satellite $(m)$, or not.

Tasks' constraints determine the completion time of a specific sequence on a specific satellite, which is equal to the sum of the starting time and the working time of the 
current sequence and no larger than the available window end time of the task processed in the current sequence. This constraint can be represented by Eqns. (30), (31) and (32).

$$
\begin{aligned}
& S_{b m}+P_{b m}=C_{b m} \\
& \sum_{j=1}^{n} \sum_{s=1}^{w} E\left(w_{j s m}\right) \cdot x_{j b s m} \geq C_{b m} \\
& t_{e i}-t_{s i} \geq d_{i}
\end{aligned}
$$

where:

$j \ldots .$. Task index, $j=\{1,2, \ldots \ldots ., n\}$

$b$.... Sequence index, $b=\{1,2, \ldots \ldots ., n\}$

$s \ldots$. Window index, $s=\{1,2, \ldots \ldots ., w\}$

$m$.... Satellite index, $m=\{1,2, \ldots \ldots . ., k\}$

$S_{b m} \ldots$. The starting time of the $b^{t h}$ sequence scheduled on satellite $(m)$

$P_{b m} \ldots$. The working time of the $b^{\text {th }}$ sequence scheduled on satellite $(m)$

$C_{b m} \ldots$. The completion time of the $b^{\text {th }}$ sequence scheduled on satellite $(m)$

$w_{j s m} \ldots$. The $s^{\text {th }}$ time window of task $(j)$ on satellite $(m)$

$E\left(w_{j s m}\right) \ldots$. The end time of time window

$x_{j b s m} \ldots$. A decision/Boolean variable representing if task $(j)$ is assigned at the $s^{\text {th }}$ window index with the $b^{\text {th }}$ sequence scheduled on satellite $(m)$, or not.

$t_{e i} \ldots$. The ending time for observing task $(i)$

$t_{s i} \ldots$. The starting time for observing task $(i)$

$d_{i} \ldots$ Continuous observation time

Tasks' constraints ensure that each available time window is longer than the shortest running time of its observing satellite and shorter than the longest running time of the satellite; this constraint can be represented by Eqn. (33).

$x_{i k}^{j} t_{\min j} \leq x_{i k}^{j}\left(e t_{i k}^{j}-s t_{i k}^{j}\right) \leq x_{i k}^{j} t_{\max j}$

where:

$T$.... Set of tasks, $\forall T=\{1, \ldots ., n\}, \forall i \in T$

$S$.... The set of satellites, $\forall j \in S$

$W_{i}{ }^{j} \ldots$. The set of time windows observed by satellite $(j)$ for task $(i), \forall k \in W_{i}^{j}$

$x_{i k}^{j} \ldots$. The decision/Boolean variable representing if the task $(i)$ can be observed by satellite $(j)$ in the $k^{\text {th }}$ time window or not

$t_{\min j} \ldots$. The shortest running time for satellite $(j)$

$t_{\max j} \ldots$. The longest running time for satellite $(j)$

$s t_{i k}^{j} \ldots$. The start time the $k^{\text {th }}$ time window observed by satellite $(j)$ for task $(i)$ 
$e t_{i k}^{j} \ldots$. The end time the $k^{\text {th }}$ time window observed by satellite $(j)$ for task $(i)$

\section{Scheduling}

Tasks' constraints ensure that each task can only be scheduled to the orbits that are available for it [21]. This constraint can be represented by Eqns. (34) and (35).

$$
\begin{aligned}
& \sum_{\substack{j \in T \\
i \neq j}} \cup\{n+1\} \\
& x_{i k}^{k} \leq b_{i k} \\
& { }_{i k}
\end{aligned}
$$

where:

$T$.... Set of tasks, $\forall T=\{1, \ldots, n\}, \forall i \in T$

$i, j \ldots$. Task index, $i, j \in T \cup\{0, n+1\}$ is the immediate predecessor of task $(j)$

$O$.... Set of orbits, $\forall O=\{1, \ldots ., m\}$

$k$.... Orbit index, $\forall k \in O$

$x_{i j}^{k} \ldots$ Decision variable, $x_{i j}^{k}=1$ If both tasks $(i),(j)$ are scheduled on orbit $(k)$ and task $(i)$ is the immediate predecessor of task $(j)$; Otherwise $x_{i j}^{k}=0$

$x_{i k} \ldots$ A Decision variable, $x_{i k}=1$ If both tasks $(i)$ is allocated to orbit $(k)$; Otherwise $x_{i k}=0$

$b_{i k} \ldots$ The binary variable representing if task $(i)$ can be observed by on orbit $(k)$ or not

Tasks' constraints ensure that each task can be processed in no more than one window with one sequence on one satellite [3]. This constraint can be represented by Eqn. (36).

$$
\sum_{m=1}^{k} \sum_{s=1}^{w} \sum_{b=1}^{n} x_{j b s m} \leq 1
$$

where:

$j \ldots$. Task index $j=\{1,2, \ldots \ldots ., n\}$

$b$.... Sequence index, $b=\{1,2, \ldots \ldots, n\}$

$s \ldots$. Window index, $s=\{1,2, \ldots \ldots ., w\}$

$m$.... Satellite index, $m=\{1,2, \ldots \ldots ., k\}$

$x_{j b s m} \ldots$. A decision/Boolean variable representing if task $(j)$ is assigned at the $s^{\text {th }}$ window index with the $b^{\text {th }}$ sequence scheduled on satellite $(m)$, or not.

It ensures that each sequence on one satellite can be assigned no more than one task in one window [3, 23]. This constraint can be represented by Eqn. (37).

$$
\sum_{j=1}^{n} \sum_{s=1}^{w} x_{j b s m} \leq 1
$$


where:

$j \ldots$. Task index,$j=\{1,2, \ldots \ldots ., n\}$

$b$.... Sequence index, $b=\{1,2, \ldots \ldots, n\}$

$s$.... Window index, $s=\{1,2, \ldots \ldots ., w\}$

$m$.... Satellite index, $m=\{1,2, \ldots \ldots ., k\}$

$x_{j b s m} \ldots$. A decision/Boolean variable representing if task $(j)$ is assigned at the $s^{\text {th }}$ window index with the $b^{\text {th }}$ sequence scheduled on satellite $(m)$, or not.

\section{Consecutive observations}

It ensures that there is a sufficient setup time for transformation between consecutive tasks [4, 12, 21, 26, 28, and 29]. This constraint can be represented by Eqn. (38).

$$
x_{i j}^{k}\left(\omega s_{j k}-\omega e_{i k}-s t_{i j}^{k}\right) \geq 0
$$

where:

$T$... Set of tasks, $\forall T=\{1, \ldots, n\}, \forall i \in T$

$i, j \ldots$. Task index, $i, j \in T \cup\{0, n+1\}$ is the immediate predecessor of $\operatorname{task}(j)$

$O$.... Set of orbits, $\forall O=\{1, \ldots ., m\}$

$k$.... Orbit index, $\forall k \in O$

$s t_{i j}^{k} \ldots$. The setup time between task $(i)$ and task $(j)$ on orbit $(k)$

$x_{i j}^{k} \ldots$ Decision variable, $x_{i j}^{k}=1$ If both tasks $(i),(j)$ are scheduled on orbit $(k)$ and task $(i)$ is the immediate predecessor of task $(j)$; Otherwise $x_{i j}^{k}=0$

It ensures that any two observation time windows satisfy the switch time requirement [20]. This constraint can be represented by Eqn. (39).

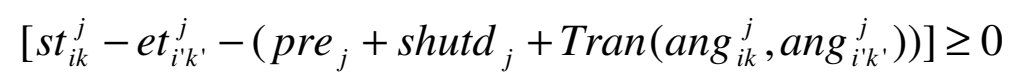

where:

$s t_{i k}^{j} \ldots$. The start time the $k^{\text {th }}$ time window observed by satellite $(j)$ for task $(i)$

$e t_{i^{\prime} k^{\prime}}^{j} \ldots$. The end time the $k^{\text {th }}$ time window observed by satellite $(j)$ for task $\left(i^{\prime}\right)$

pre $_{j} \ldots$. The set up time of satellite $(j)$ for starting

shutd $_{j} \ldots$... The stable time of satellite $(j)$ shutdown

$\operatorname{Tran}\left(a n g_{i k}^{j}, a^{\prime} g_{i^{\prime} k^{\prime}}^{j}\right) \ldots$. The switching time that the same satellite $(j)$ adjusts its swing angle from ang $_{i k}^{j}$ to ang $_{i^{\prime} k^{\prime}}^{j}$ for completing task $\left(i^{\prime}\right)$ after having completed task $(i)$ when task $(i)$ and task $\left(i^{\prime}\right)$ are adjacent

$x_{i k}^{j} \ldots$. The decision/Boolean variable representing if the task $(i)$ can be observed by satellite $(j)$ in the $k^{\text {th }}$ time window or not

$x_{i^{\prime} k^{\prime}}^{j} \ldots$. The decision/Boolean variable representing if the task $\left(i^{\prime}\right)$ can be observed by satellite $(j)$ in the $k^{\text {th }}$ time window or not 


\section{Ground station}

\section{Range of ground station}

Downloading is only allowed if the satellite in the range of the ground station [7]; this constraint can be represented by Eqn. (40).

$$
x_{s i g} \leq \gamma_{s i g}
$$

where:

$I \ldots$. Set of intervals, $\forall i \in I$

$S$.... Set of satellites, $\forall s \in S$

$G$.... Set of ground stations, $\forall g \in G$

$x_{s i g} \ldots$. The continuous variable representing the percentage of interval $(i)$ during which satellite $(s)$ downloads to ground station $(g), x_{\text {sig }} \in[0,1]$

$\gamma_{\text {sig }} \ldots$. The binary variable representing if the satellite $(s)$ is in view of ground station $(g)$ during interval $(i)$

\section{Time interval for ground station}

Each ground station cannot receive/transmit data for more than $100 \%$ of each time interval [7, 26]. This constraint can be represented by Eqns. (41) and (42).

$$
\begin{aligned}
& \sum_{s \in S} x_{s i g} \leq 1 \\
& \sum_{i \in G} x_{s i g} \leq 1
\end{aligned}
$$

where:

$I \ldots$ Set of intervals, $\forall i \in I$

$S$.... Set of satellites, $\forall s \in S$

$G$.... Set of ground stations, $\forall g \in G$

$x_{s i g} \ldots$. The continuous variable representing the percentage of interval $(i)$ during which satellite $(s)$ downloads to ground station $(g), x_{s i g} \in[0,1]$

\section{Capacity of ground station}

Each ground station can process without preemption at most one service at a specific time [23]. This constraint can be represented by Eqn. (43).

$$
\sum_{j=1}^{J} \sum_{\tau \in \Theta(j, m, t)} x_{j m}^{\tau} \leq 1
$$

where:

$\tau \ldots$. Set of services, $\forall j \in \tau, \tau=\{1, \ldots . . J\}$

$x_{j m}^{\tau} \ldots$. Binary variable, $x_{j m}^{\tau}=1$ If the ground station $(m)$ starts processing service $(j)$ at time $(t)$ 


\section{OPTIMIZATION METHODS}

For solving planning and scheduling problem for earth observation satellites, many optimization methods have been used in many papers.

\section{Methods of Optimization}

There are many methods of optimization such as:

- Dynamic Programming (DP)

- Simulated Annealing (SA)

- Look Ahead Algorithm (L.A. Alg.)

- Constraint Programming Approach (CPA)

- Local Search Method (LS Method)

- Branch and price algorithm (BP Alg.)

- Branch and cut algorithm (BC Alg.)

- Ant colony optimization (ACO)

- Genetic algorithm (GA)

- Heuristic algorithm (H. Alg.)

- Priority-Based heuristic algorithm (PB H. Alg.)

- Metaheuristic-Based Approach

- Linear Programming (LP)

- Greedy algorithm (Gr. Alg.)

- Evolutionary algorithm (Ev. Alg.)

- Dynamic scheduling (DS)

\section{The Relation between Objective Functions, Constraints and Methods of Optimization}

After surveying many papers [References 7-31], Table 1 could be a good method and easy way to explain the combined satellite optimization problem objective functions, constraints and optimization's methods.

From Table 1, the following points are deduced:

- A gap exists between objectives which are relative to data and the targets' constraints so it is considered to be logic because there is no need to include targets' constraints with data's objectives.

- The most widely used optimization methods are dynamic programming and heuristic algorithms.

- Dynamic programming has a widely used because of many reasons; it is one of the elegant algorithm design standards, it is a powerful tool that yields classic algorithms for a variety of combinatorial optimization problems, it can be considered as a useful first approximation scheme to human decision making and its simplicity appear because of a full problem solving method and a subroutine solver in more complicated algorithmic solutions. 
- Heuristic algorithms have a widely used because of they are usually find a solution close to the best one and they find it fast and easily. Sometimes these algorithms can be accurate, that is they actually find the best solution, but the algorithm is still called heuristic until this best solution is proven to be the best.

- The most widely used objectives are those relative to profit and it seems to be logic as profit is considered an important objective for everyone and every organization.

- The constraints which involve data rate, range of ground stations and capacity of ground stations have a few discussions. The reason for few discussions may be that researchers gave an attention for data buffer limits/storage capacity more than data rate and for intervals time of ground stations more than those capacity and range.

- Iterative algorithm has been used as an optimization method just between data's objectives and constraints of energy and data. Where, it limits the constraints to converge to a feasible and thus optimal solution and also introduces new variables and constraints and solves a newly larger defined problem at each iteration so it can conceptualize as capturing computation in a set of state variables which update on each iteration and it depends mainly on looping statements.

- Sample approximation and simulated annealing have been used as optimization methods between profit's objectives and constraints of energy, data and tasks/targets. Where, Sample approximation transforms the chance constraint programming (CCP) model into an integer linear programming (ILP) model and simulated annealing (SA) has better global search ability. Some people improved SA and proposed a very fast simulated annealing (VFSA) greatly increased the computation speed. It has been used with an ant colony optimization (ACO) to make an adaptive ACO which is better than ACO only.It is important to mention that simulated annealing is used with one of other optimization algorithms together as hybrids algorithms for global optimization as simulated annealing is used to find a rough estimate of the solution and then the other optimization algorithm is used to refine the solution.

- Evolutionary algorithm, constraint programming approach (CPA) and local search method (LS Method) have been used as optimization methods just between profit's objectives and target's constraints. Where,Strength Pareto evolutionary algorithm (SPEA) has shown better performance in comparison with other multiobjective evolutionary algorithms. CPA \& LSMethod were able to take all real specific operational constraints in consideration. CPA is very flexible while LS gives better performance and easy to be understood.

- M. Lemaître et al. have discussed CPA and LS method for AEOS Track Selection and Scheduling problem. CPA used some heuristics to add constraints to the model; eliminate images having very small weights, limiting backtracks, that are a transition between two images going in the opposite movement of the satellite, even the agility of the satellite permit them. While using LS method, a current feasible solution sequence of images is maintained and a neighbouring solution is obtained by inserting or removing an image in or from the current sequence. 
- Priority based, priority based conflict avoided are types of heuristic algorithms and all of them have been used as optimization methods. Where, priority based and priority based conflict avoided produce satisfactory feasible plans in a very short time.

- The Look Ahead algorithm and genetic algorithm have been used as optimization methods. Where, The Look Ahead algorithm runs a little slower but is still relatively simple, creates excellent schedules and provides significant improvements in quality. The genetic algorithm is a lot slower but creates nearoptimal schedules and provides significant improvements on some cases.

\section{CONCLUSION}

This paper discussed the planning and scheduling problem of earth observation satellites showing the objective functions, constraints and methods of optimization which have been used in many papers.

Space system has been discussed briefly including definitions and some applications.

Objective functions and constraints have been categorized related to many factors. Objective functions, constraints and methods of optimization have been collected together in a table showing the relation between them.

It was found that the profit has been discussed too much as an important objective, dynamic programming and heuristic algorithms have been used widely which are considered optimization methods and some constraints such as data rate, range of ground stations and capacity of ground stations have few discussions. So, it will be easy now to get information about this relation.

For future research, the objective function of quality needs more researches and discussions using the suitable optimization technique. 


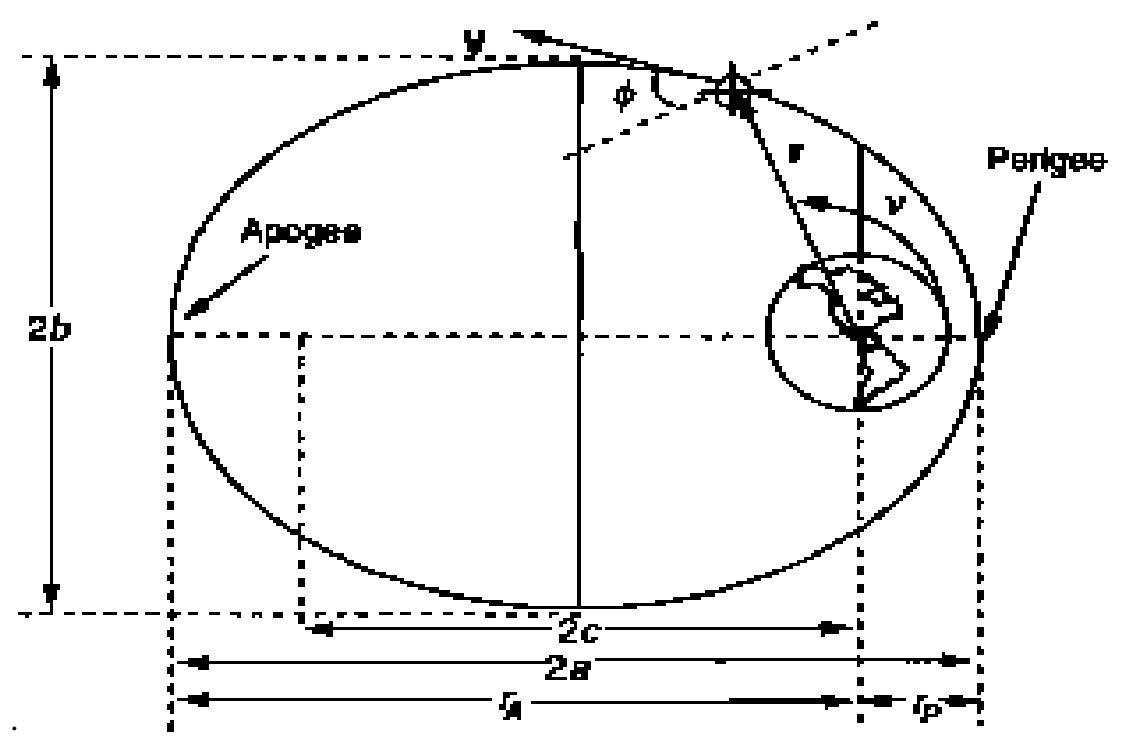

Fig.1.Parameters of elliptical orbit [16].

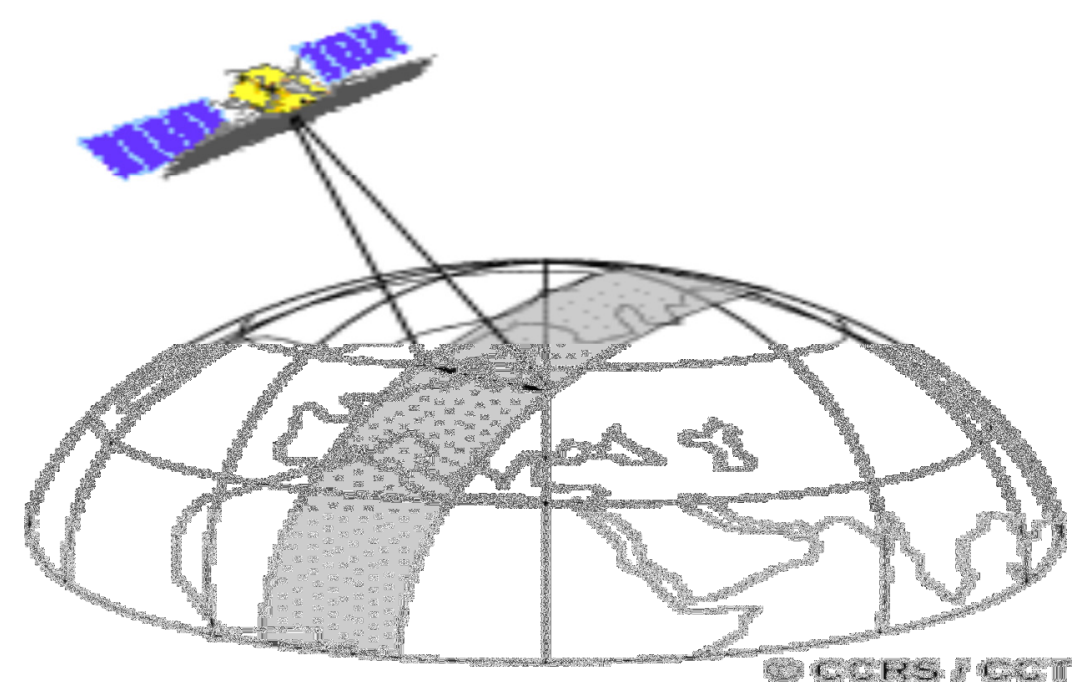

Fig.2. Schematic representation of the satellite swath [18].

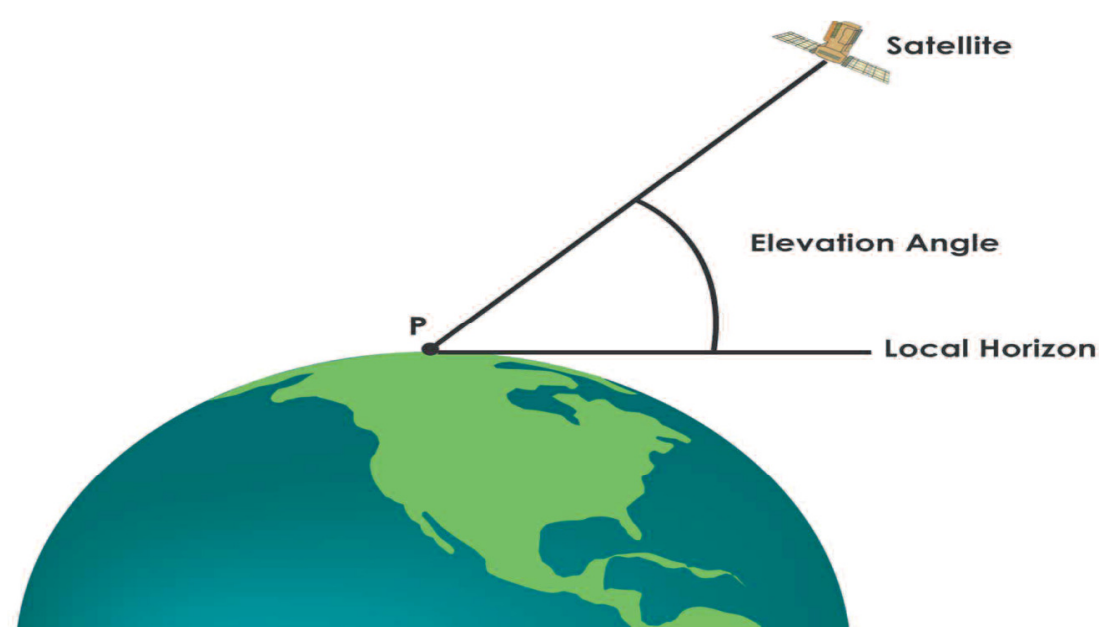

Fig.3. The elevation angle of a satellite at a given time for an observer at the point $P$ on the Earth is the angle between the local horizon at $P$ and a line from the observer to the satellite [19]. 


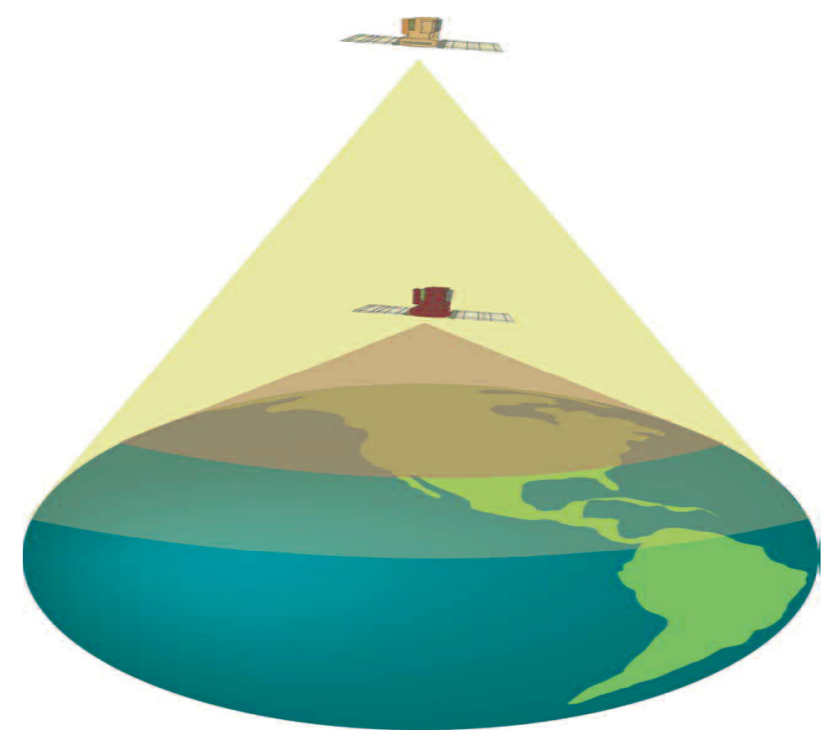

Fig.4.Two satellites at different altitude [19].

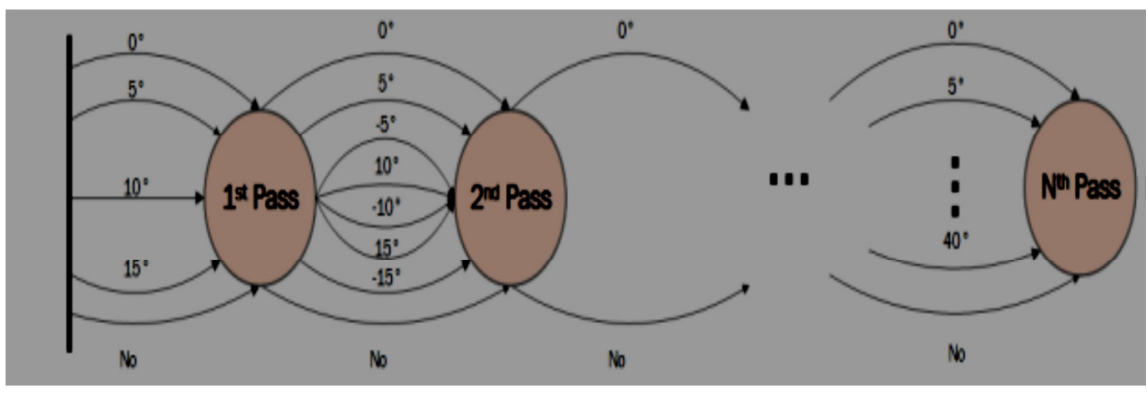

Fig.5.Problem representation using a directed graph [11]. 
Table 1. The relation between objective functions, constraints and optimization

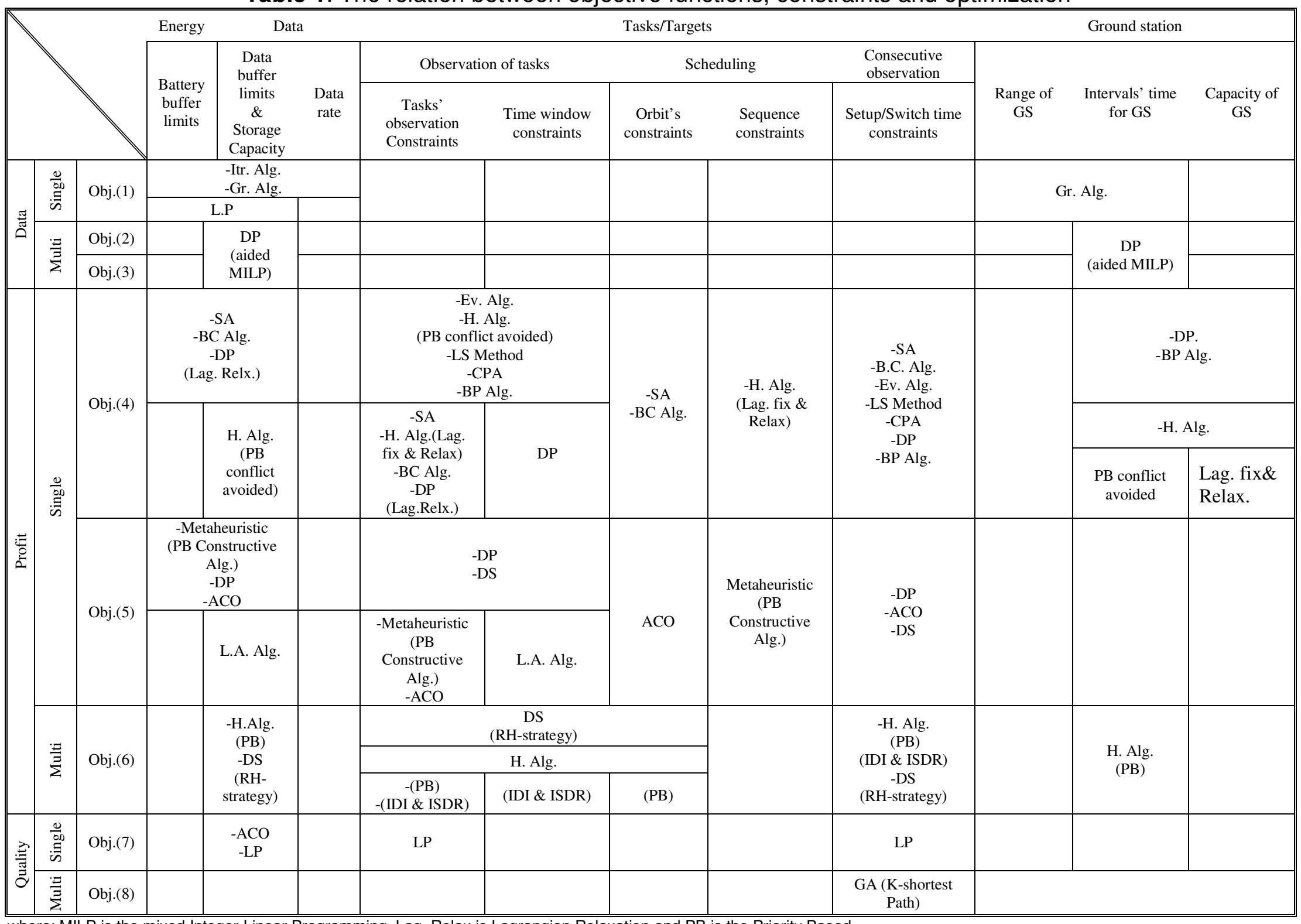


Table 2. The meaning of objectives' numbers

\begin{tabular}{|c|c|}
\hline $\begin{array}{c}\text { Objectives' } \\
\text { Number }\end{array}$ & Objectives Functions \\
\hline 1 & Maximize the total amount of downloaded data \\
\hline 2 & $\begin{array}{c}\text { Collecting the largest amount of prioritized imagery and } \\
\text { continually lowering the amount of un-downloaded data onboard } \\
\text { the satellite }\end{array}$ \\
\hline 3 & $\begin{array}{c}\text { Not miss out on imagery and continually lowering the amount of } \\
\text { un-downloaded data onboard the satellite }\end{array}$ \\
\hline 4 & Maximize the profits of the scheduled tasks \\
\hline 5 & Maximize the total priority of selected tasks \\
\hline 6 & Completing dynamic tasks as many as possible and maximize \\
& the observation income \\
\hline 7 & Maximize quality values \\
\hline 8 & Maximize weight value \\
\hline
\end{tabular}

\section{REFERENCES}

[1] Li, J., H. Chen, and N. Jing, A data transmission scheduling algorithm for rapidresponse earth-observing operations. Chinese Journal of Aeronautics, 2014. 27(2): p. 349-364.

[2] Kai Sun1, Jufang Li2, Yingwu Chen3 and Renjie He4, Multi-objective mission planning problem of agile Earth observing satellites. 2012.

[3] $\mathrm{R} \mathrm{Xu}, \mathrm{H}$ Chen, X Liang and $\mathrm{H}$ Wang, Priority-based constructive algorithms for scheduling agile earth observation satellites with total priority maximization. Expert Systems with Applications, 2016. 51: p. 195-206.

[4] Michel Lemaître, Gérard Verfaillie, Frank Jouhaud, Jean-Michel Lachiver and Nicolas Bataille, Selecting and scheduling observations of agile satellites. Aerospace Science and Technology, 2002. 6(5): p. 367-381.

[5] Liu Xiaolu, Bai Baocun, Chen Yingwu and Yao Feng, Multi satellites scheduling algorithm based on task merging mechanism. Applied Mathematics and Computation, 2014. 230: p. 687-700.

[6] Sara Spangeloa, James Cutlera, Kyle Gilsonb and Amy Cohn, Optimizationbased scheduling for the single-satellite, multi-ground station communication problem. Computers \& Operations Research, 2015. 57: p. 1-16.

[7] Castaing, J., Scheduling Downloads for Multi-Satellite, Multi-Ground Station Missions. 2014.

[8] Sara Spangelo and James Cutler, Optimization of single satellite operational schedules towards enhanced communication capacity. In American Institute of Aeronautics and Astronautics Guidance, Navigation and Control Conference, 13-16 August 2012, Minnesota. p. 1-14.

[9] Sean Augenstein, Alejandra Estanislao, Emmanuel Guere and Sean Blaes. Optimal Scheduling of a Constellation of Earth-Imaging Satellites, for Maximal Data Throughput and Efficient Human Management. In Twenty-Sixth International Conference on Automated Planning and Scheduling. 2016.

[10] Liu Xiaolu, Bai Baocun, Chen Yingwu and Yao Feng, Multi satellites scheduling algorithm based on task merging mechanism. Applied Mathematics and Computation, 2014. 230: p. 687-700. 
[11] Evridiki Vasileia Ntagiou Automated Planning and Scheduling EO Constellations' Operations with Ant Colony Optimization. University of Surrey. In Twenty-Sixth International Conference on Automated Planning and Scheduling. 2016.

[12] Wang, J., E. Demeulemeester, and D. Qiu, A pure proactive scheduling algorithm for multiple earth observation satellites under uncertainties of clouds. Computers \& Operations Research, 2016. 74: p. 1-13.

[13] C. lacopino, P. Palmer, N. Policella, A. Donati and A. Brewer, Self-Organizing MPS for Dynamic EO Constellation Scenarios. Proc. IWPSS, 2013.

[14] https://www.nasa.gov/audience/forstudents/5-8/features/nasa-knows/what-is-asatellite-58.html Jan. 10, 2017.

[15] http://archive.mu.ac.in/myweb test/Satelight\%20Comm..pdf Jan. 10, 2017.

[16] Space mission analysis and design , Wiley J. Larson.

[17] http://www.g4ztd.info/wp-content/uploads/2012/09/howsatellitework.pdf Jan. 10,2017

[18] D. Nagesh Kumar, Satellites and orbits. National Program on Technology Enhanced Learning (NPTEL), Module - 2, Lecture Notes - 1. p. 1-9, 2007.

[19] David Wright, Laura Grego and Lisbeth Gronlund, The physics of space security (A reference manual).American Academy of Arts and Sciences, 2005. p. 29-48.

[20] Wang, M., G. Dai, and M. Vasile, Heuristic scheduling algorithm oriented dynamic tasks for imaging satellites. Mathematical Problems in Engineering, 2014. 2014.

[21] Wang, J., E. Demeulemeester, and D. Qiu, A pure proactive scheduling algorithm for multiple earth observation satellites under uncertainties of clouds. Computers \& Operations Research, 2016. 74: p. 1-13.

[22] Qiu Dishan, He Chuan, Liu Jin, and Ma Manhao, A dynamic scheduling method of earth-observing satellites by employing rolling horizon strategy. The Scientific World Journal, 2013.

[23] Fabrizio Marinelli, Salvatore Nocella, Fabrizio Rossi and Stefano Smriglio, A Lagrangian heuristic for satellite range scheduling with resource constraints. Computers \& Operations Research, 2011. 38(11): p. 1572-1583.

[24] Bianchessi, N. and G. Righini. A mathematical programming algorithm for planning and scheduling an earth observing SAR constellation. In Proceeding of the fifth international workshop on planning and scheduling for space (IWPSS'06). 2006. Citeseer.

[25] Guohua Wu, Jin Liu, Manhao Ma, Dishan Qiu, A two-phase scheduling method with the consideration of task clustering for earth observing satellites. Computers \& Operations Research, 2013. 40(7): p. 1884-1894.

[26] Wang, P. and G. Reinelt, A heuristic for an earth observing satellite constellation scheduling problem with download considerations. Electronic Notes in Discrete Mathematics, 2010. 36: p. 711-718.

[27] Jianjiang Wang, Xiaomin Zhu, Dishan Qiu and Laurence T. Yang, Dynamic scheduling for emergency tasks on distributed imaging satellites with task merging. IEEE Transactions on Parallel and Distributed Systems, 2014. 25(9): p. 2275-2285.

[28] Feng Yao, Jufang Li, Baocun Bai and Renjie He, Earth observation satellites scheduling based on decomposition optimization algorithm. International Journal of Image, Graphics and Signal Processing, 2010. 2(1): p. 10.

[29] Wang, P. and G. Reinelt, Solving the Earth Observing Satellite Constellation Scheduling Problem by Branch-and-Price, in Operations Research Proceedings 2010. 2011, Springer. p. 491-496. 
[30] Gabrel, V., Strengthened 0-1 linear formulation for the daily satellite mission planning. Journal of combinatorial optimization, 2006. 11(3): p. 341-346.

[31] Wolfe, W.J. and S.E. Sorensen, Three scheduling algorithms applied to the earth observing systems domain. Management Science, 2000. 46(1): p. 148166. 